\title{
Correlation between the 2-Dimensional Extent of Orbital Defects and the 3-Dimensional Volume of Herniated Orbital Content in Patients with Isolated Orbital Wall Fractures
}

\author{
Jong Hyun $\mathrm{Cha}^{1}$, Myeong Ho Moon ${ }^{2}$, Yong Hae Lee ${ }^{1}$, In Chang $\mathrm{Koh}^{1}$, Kyu Nam Kim ${ }^{1}$, \\ Chang Gyun Kim ${ }^{1}$, Hoon Kim ${ }^{1}$ \\ ${ }^{1}$ Department of Plastic and Reconstructive Surgery, Konyang University Hospital, University of Konyang College of Medicine, Myunggok \\ Medical Research Center, Daejeon; ${ }^{2}$ Biomedical Engineering, Konyang University College of Medicine, Daejeon, Korea
}

Background The purpose of this study was to assess the correlation between the 2-dimensional (2D) extent of orbital defects and the 3-dimensional (3D) volume of herniated orbital content in patients with an orbital wall fracture.

Methods This retrospective study was based on the medical records and radiologic data of 60 patients from January 2014 to June 2016 for a unilateral isolated orbital wall fracture. They were classified into 2 groups depending on whether the fracture involved the inferior wall (group I, $n=30$ ) or the medial wall (group $M, n=30$ ). The $2 D$ area of the orbital defect was calculated using the conventional formula. The 2D extent of the orbital defect and the 3D volume of herniated orbital content were measured with 3D image processing software. Statistical analysis was performed to evaluate the correlations between the 2D and 3D parameters.

Results Varying degrees of positive correlation were found between the 2D extent of the orbital defects and the 3D herniated orbital volume in both groups (Pearson correlation coefficient, $0.568-0.788 ; R^{2}=32.2 \%-62.1 \%$ ).

Conclusions Both the calculated and measured 2D extent of the orbital defects showed a positive correlation with the $3 \mathrm{D}$ herniated orbital volume in orbital wall fractures. However, a relatively large volume of herniation $\left(>0.9 \mathrm{~cm}^{3}\right)$ occurred not infrequently despite the presence of a small orbital defect $\left(<1.9 \mathrm{~cm}^{2}\right)$. Therefore, estimating the 3D volume of the herniated content in addition to the $2 \mathrm{D}$ orbital defect would be helpful for determining whether surgery is indicated and ensuring adequate surgical outcomes.

Keywords Imaging, three-dimensional / Orbital fractures / Orbit

\author{
Correspondence: Hoon Kim \\ Department of Plastic and \\ Reconstructive Surgery, Konyang \\ University Hospital, University of \\ Konyang College of Medicine, \\ Myunggok Medical Research Center, \\ 158 Gwanjeodong-ro, Seo-gu, \\ Daejeon 35365, Korea \\ Tel: $+82-42-600-9210$ \\ Fax: +82-42-600-9090 \\ E-mail: prshoonkim@gmail.com
}

No potential conflict of interest relevant to this article was reported.

\section{INTRODUCTION}

Isolated orbital fracture-also known as pure blow-out frac- ture-is one of the most commonly encountered types of facial fracture because of the vulnerability of the orbit to external impacts, as well as the structural weakness of the thin bony layers 
[1]. These fractures may cause volumetric expansion of the orbital cavity and a shift of the intraorbital contents, resulting in diplopia, restricted eye movement, disturbances in visual acuity, and enophthalmos [1-5].

The conventional surgical criteria for open reduction followed by orbital reconstruction in cases of orbital fractures are the presence of the following symptoms and signs: diplopia with limitation of extraocular movement due to muscle entrapment, radiologic evidence of extensive fracture, and significant enophthal$\operatorname{mos}[2,6]$. Among these surgical indications, the possible development of late enophthalmos has been regarded as one of the most significant factors favoring surgical intervention $[1,7,8]$.

Therefore, numerous trials have been conducted to predict the degree of enophthalmos based on the 2-dimensional (2D) orbital defect area or the 3-dimensional (3D) expanded volume of the orbit $[2,5,8-11]$.

Previous studies have revealed that the size of the calculated or measured 2D orbital defect on a preoperative $\mathrm{CT}$ scan was predictive of subsequent enophthalmos. Manson et al. [2] elucidated that an orbital defect over $2 \mathrm{~cm}^{2}$ could lead to a significant posterior globe displacement of more than $3 \mathrm{~mm}$. Volumetric analyses have demonstrated that a $5 \%$ increase in the total orbital volume can be considered sufficient to result in clinically significant enophthalmos $[2,10]$.

Recently, a novel 3D software tool providing a practical way to measure the orbital defect area and 3D volume was introduced to translate stacks of $2 \mathrm{D}$ image data created by scanning systems into 3D objects [12,13].

However, to the best of our knowledge, no reports have evaluated the correlations between $2 \mathrm{D}$ and $3 \mathrm{D}$ parameters in orbital wall fractures using $3 \mathrm{D}$ image processing software. Herein, we provide a statistical analysis of the correlations between the calculated and measured 2D extent of orbital defects and the 3D volume measurements of the herniated orbital content.

\section{METHODS}

In this retrospective study, 60 patients with unilateral isolated orbital wall fractures from January 2014 to June 2016 were investigated using radiologic and medical records. Patients with combined fractures that could potentially influence the volume of the orbit, such as zygomaticomaxillary fractures, frontal sinus fractures involving the orbital roof, and bilateral orbital bone fractures were excluded. The subjects were divided into 2 groups: group I $(\mathrm{n}=30)$ included patients with an inferior blowout fracture and group $M(n=30)$ included patients with a medial blow-out fracture.

The calculated 2D extent of the orbital defect, the measured
$2 \mathrm{D}$ area of the orbital defect, and the 3D volume of the herniated orbital content protruding through the orbital defect were estimated. The calculated 2D orbital defect was defined as the area obtained using the conventional formula [8]. The measured 2D orbital defect was defined as the surface area measured by computer-aided design (CAD) software capable of tracing the boundary of the orbital defect in a $3 \mathrm{D}$ rendering model. The estimated 3D herniated orbital volume was defined as the 3D volumetric value of the herniated orbital content obtained using $3 \mathrm{D}$ processing software.

Statistical analysis was performed to identify the correlations among the calculated 2D defect, the measured 2D defect, and the $3 \mathrm{D}$ volume of the herniated orbital content.

\section{Computed tomography scans and 3D model rendering} Targeted axial and coronal sections were obtained from the raw data generated by computed tomography (CT) scans (SOMATOM definition flash; SIEMENS, Munich, Germany). The CT scans were evaluated at the time of trauma. The mean interval from the time of trauma was $0.32 \pm 0.93$ days. The CT data were converted to the Digital Imaging and Communications in Medicine format and transferred to the Department of Biomedical Engineering. Image processing and 3D rendering was performed in Mimics ver. 17.0 (Materialise, Leuven, Belgium). For the $3 \mathrm{D}$ rendering, segmentation of the CT scan was performed, in which specific elements were separated and structures outside of the scope of the current study were removed. Based on the specific values of Hounsfield units (HU) for organic substances, the bony orbit and orbital soft tissue were selected semiautomatically using the threshold interval tool in the software (1,000 HU for bone and -100 to $-50 \mathrm{HU}$ for orbital fat) [14]. After manual editing of the segmentation was performed to reduce the presence of artifacts, the 3D-rendered orbital and herniated orbital volume models from the coronal and axial planes were fused via CAD software (3-matic Research 9.0, Materialise) using the same anatomical landmarks.

\section{Calculated 2D orbital defect}

The 2D defect of the orbital wall seen on this targeted sectional view was calculated using the conventional formula [8]. The major axis was defined as the distance between the anterior and posterior ends of the defect, and the minor axis was defined as the distance between the medial and lateral ends. The boundary of the defect was confirmed on the fused 3D orbital model, and the length was obtained using the measurement tool in the CAD software package (3-matic Research 9.0, Materialise) (Fig. $1 \mathrm{~A}, \mathrm{~B})$. 


\section{Fig. 1. Calculated and measured 2D extent of defects}

The 2-dimensional (2D) extent of orbital defects was calculated using the conventional formula in group I (A) and group M (B). The major axis length and minor axis length were measured using the 3-dimensional measurement tool in a computer-aided design (CAD) software package (3-matic Research 9.0, Materialise). The 2D extent of the orbital defect was measured using the virtual surface (yellow) in an automated process in the CAD program in group I (C) and group M (D). The boundary of the defect was confirmed in various viewing directions by manually controlled rotation.
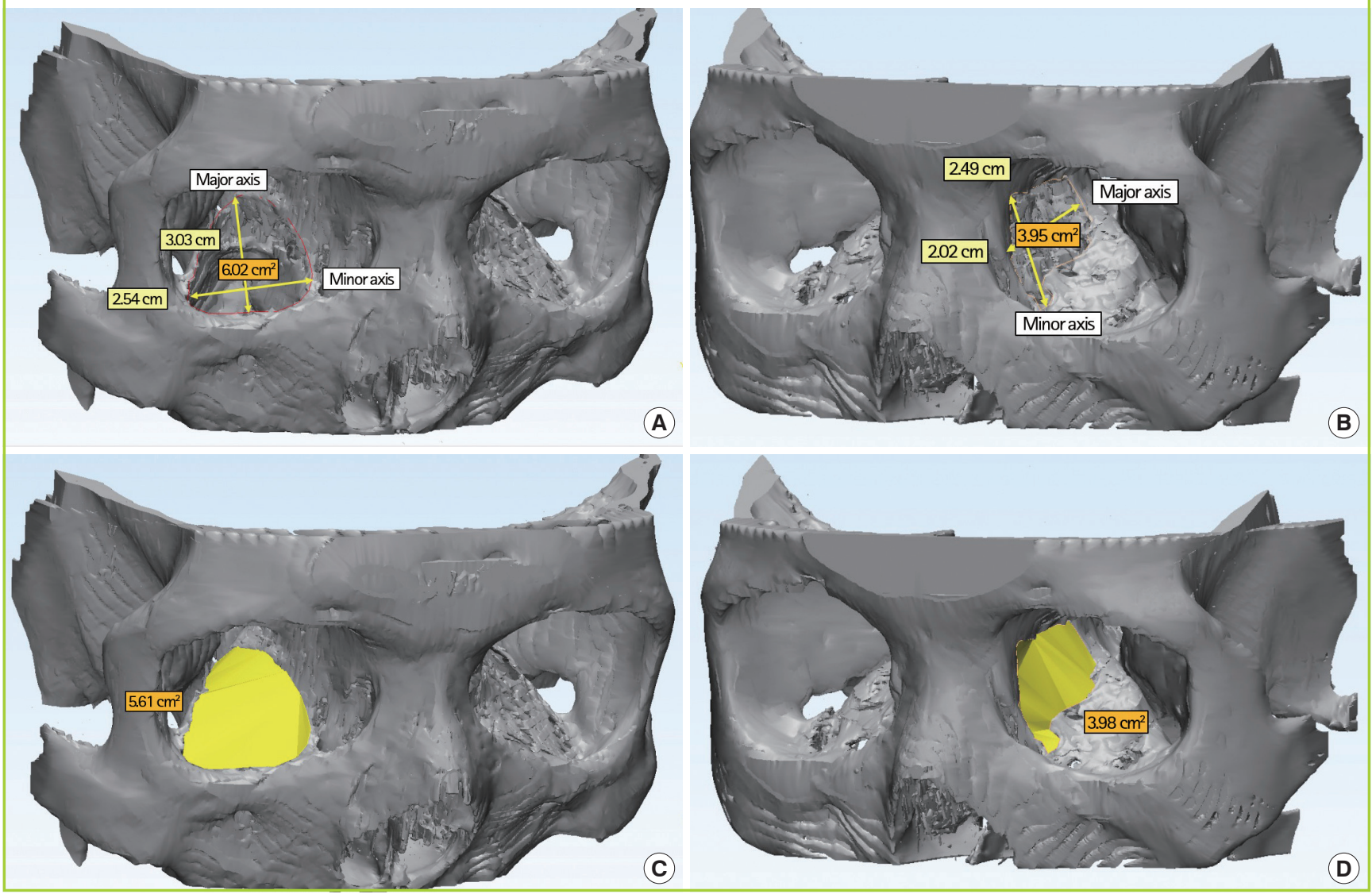

Calculated $2 \mathrm{D}$ defect $=\pi \times$ the length of the major axis $\times$ the length of the minor axis $\times 1 / 4$

\section{Measured 2D orbital defect and estimated 3D orbital volume using $3 \mathrm{D}$ image processing software}

The measured 2D orbital defect was determined using CAD software (3-matic Research 9.0, Materialise) to analyze the fused 3D rendering orbital model. The defect area was visualized in various viewing directions by manually controlled rotation of the 3D orbital rendering model. The boundary of the defect area was marked manually in accordance with the appropriate $\mathrm{HU}$ levels, and a virtual surface was made along the defect boundary via CAD software (3-matic Research 9.0, Materialise). The size of the measured $2 \mathrm{D}$ extent of the orbital defect was automatically obtained via pixel counting within the software package (Fig. 1C, D) [15].

The 3D volume of the herniated orbital content was estimated using the standard voxel gradient shading rendering technique provided in the software package. Volume was measured using segmentation that traced the herniated orbital content in both the axial and coronal sections. The boundary of the herniated orbital content was defined by a segmented bony orbit. When bone was missing on CT scans, a line was demarcated within the extent of the orbital volume, which was confirmed and separated from the hematoma semi-automatically using the $\mathrm{HU}$ threshold interval tool in the software package (Fig. 2A, B). The 3D-rendered herniated orbital content model was fused using CAD software. The area of the outline of each slice was measured, and the volume was estimated by an automated process via the $3 \mathrm{D}$ processing software. Two independent investigators, a physicist and a plastic surgeon, performed the measurements to ensure that the evaluation was objective (Fig. 2C, D).

\section{Statistical analysis}

To elucidate the correlation between the $2 \mathrm{D}$ extent of the orbital defect (calculated and measured) and the 3D volume of the 


\section{Fig. 2. Estimated herniated orbital content (3D)}

For the 3-dimensional (3D) rendering, multiplanar segmentation was performed (coronal, axial, and sagittal) in group I (A) and group M (B). The bony orbit (green) and herniated orbital soft tissue content (red) were selected using the Hounsfield unit threshold interval tool in the 3D processing software (Mimics 17.0, Materialise). The 3D rendering models with fused multiplane radiologic data derived from the CAD software (3-matic Research 9.0, Materialise) and estimated 3D herniated orbital volume were computed using automated processes in the software package (3-matic Research 9.0, Materialise) (C, D).
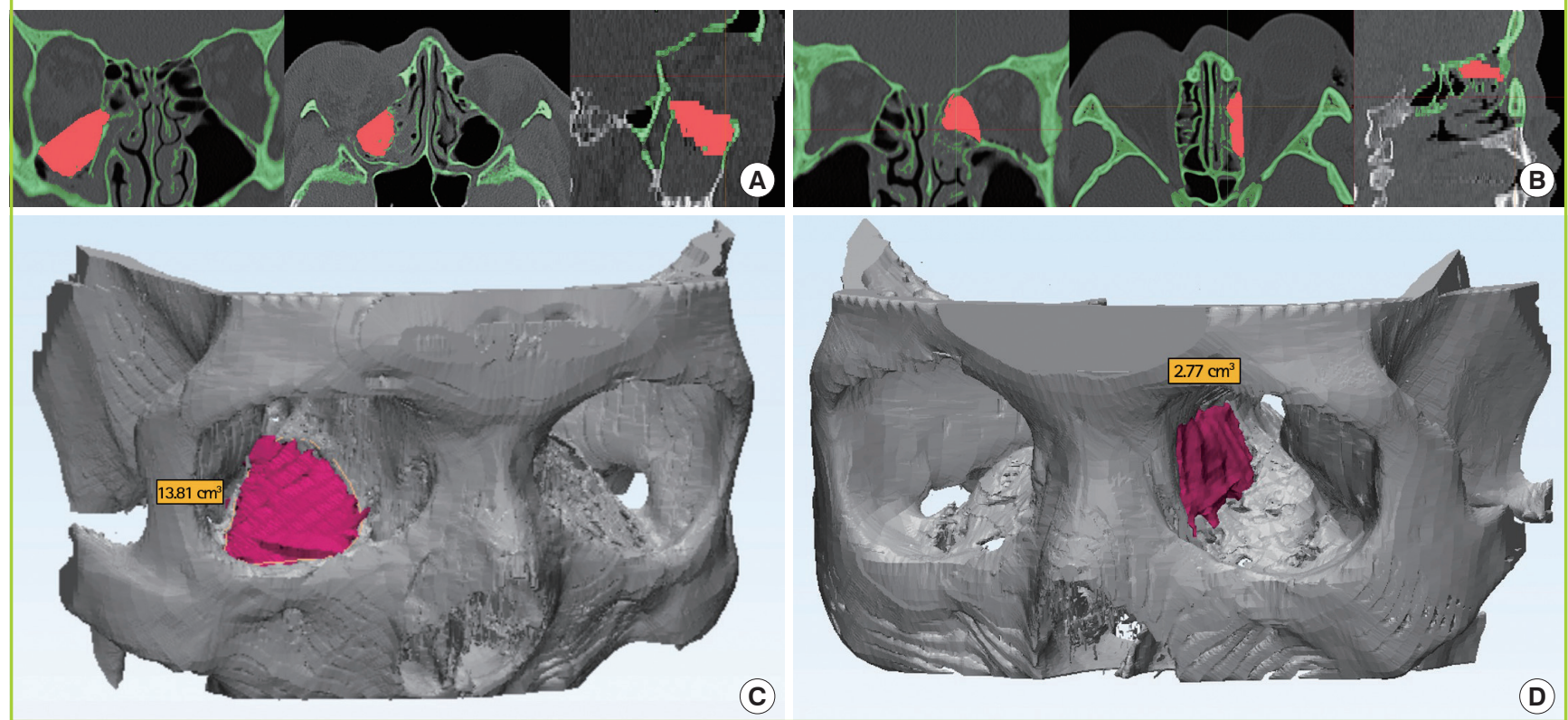

herniated orbital content protruding through the orbital defect, statistical analyses using SPSS ver. 20 (IBM Corp., Armonk, NY, USA) were performed. The normal distribution of the $2 \mathrm{D}$ parameters was confirmed using the Shapiro-Wilk test. Data were assessed for significance using the independent t-test. Correlations between the $2 \mathrm{D}$ and $3 \mathrm{D}$ variables were evaluated using the Pearson correlation coefficient. P-values $<0.05$ were considered to indicate statistical significance.

\section{RESULTS}

The mean age of patients was $40.57 \pm 18.65$ years (range, $5-81$ years). Forty-four patients were male and 16 were female. Of the patients, 42 had left-sided orbital fractures and 18 had right-sided orbital fractures.

The normal distribution of the 2D extent of the orbital defects (both calculated and measured) was confirmed using the Shapiro-Wilk test $(\mathrm{P}>0.05)$.

The average calculated $2 \mathrm{D}$ extent of the orbital defect in group I was $2.27 \pm 1.08 \mathrm{~cm}^{2}$ (range, $0.64-4.25 \mathrm{~cm}^{2}$ ), and that of the measured $2 \mathrm{D}$ area was $3.23 \pm 1.38 \mathrm{~cm}^{2}$ (range, $1.38-7.02 \mathrm{~cm}^{2}$ ). In group $\mathrm{M}$, the average calculated $2 \mathrm{D}$ extent of the orbital defect was $2.29 \pm 1.06 \mathrm{~cm}^{2}$ (range, $0.81-4.36 \mathrm{~cm}^{2}$ ), and that of the measured 2D area was $2.91 \pm 1.04 \mathrm{~cm}^{2}$ (range, $0.92-4.58 \mathrm{~cm}^{2}$ ). The calculated and measured values of the $2 \mathrm{D}$ orbital defect dif-

Table 1. Differences between the calculated and measured 2D extent of the defects

\begin{tabular}{|lccc|}
\hline \multirow{2}{*}{ Group } & \multicolumn{3}{c|}{ Extent of 2D defect $\left(\mathrm{cm}^{2}\right)$, mean \pm SD } \\
\cline { 2 - 4 } & $\begin{array}{c}\text { Calculated 2D } \\
\text { extent }\end{array}$ & $\begin{array}{c}\text { Measured 2D } \\
\text { extent }\end{array}$ & P-value ${ }^{\text {a) }}$ \\
\hline Group I $(\mathrm{n}=30)$ & $2.27 \pm 1.08$ & $3.23 \pm 1.38$ & $<0.001$ \\
Group M $(\mathrm{n}=30)$ & $2.29 \pm 1.06$ & $2.91 \pm 1.04$ & $<0.001$ \\
\hline $\begin{array}{l}\text { 2D, 2-dimensional; SD, standard deviation. } \\
\text { a)T-test, } P<0.001 .\end{array}$ & & \\
\hline
\end{tabular}

Table 2. The estimated 3D volume of the herniated orbital content

\begin{tabular}{|lc|}
\hline Group & Estimated 3D volume $\left(\mathbf{c m}^{3}\right)$, mean \pm SD \\
\hline Group I $(n=30)$ & $3.50 \pm 3.15($ range, $0.33-13.81)$ \\
Group M $(n=30)$ & $2.64 \pm 1.88$ (range, 0.37-6.64) \\
\hline 3D, 3-dimensional; SD, standard deviation. \\
\hline
\end{tabular}

fered to a statistically significant extent $(\mathrm{P}<0.001)$ (Table 1$)$.

The estimated 3D volume of the herniated orbital content in group I was $3.50 \pm 3.15 \mathrm{~cm}^{3}$ (range, $0.33-13.81 \mathrm{~cm}^{3}$ ), while in group Mit was $2.64 \pm 1.88 \mathrm{~cm}^{3}$ (range, $0.37-6.64 \mathrm{~cm}^{3}$ ) (Table 2). Jin et al. [8] concluded that 2D defects larger than $1.9 \mathrm{~cm}^{2}$ were correlated with orbital herniation volumes of $0.9 \mathrm{~cm}^{3}$, which could result in clinically significant enophthalmos (over 2 


\section{Fig. 3. Correlations between 2D and 3D parameters, group I}

In group I, the correlation between the calculated 2-dimensional (2D) extent of the defects and the estimated 3-dimensional (3D) volume (A) had an $R^{2}$-value of $62.1 \%$, while the measured $2 \mathrm{D}$ extent and 3D volume (B) had an $\mathrm{R}^{2}$-value of $54.6 \%$.
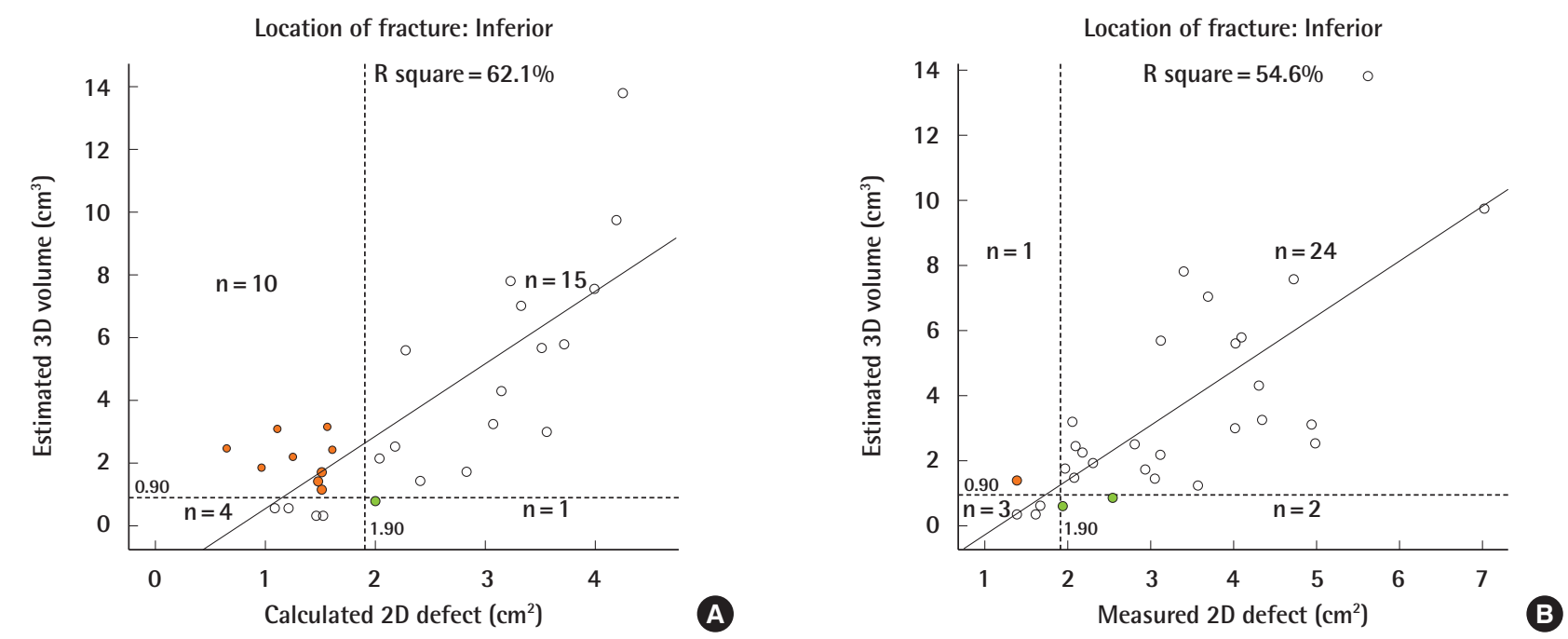

\section{Fig. 4. Correlations between 2D and 3D parameters, group M}

In group $\mathrm{M}$, the correlation between the calculated 2-dimensional (2D) extent of the defects and the estimated 3-dimensional (3D) volume (A) had an $R^{2}$-value of $32.2 \%$, while the measured $2 \mathrm{D}$ extent and $3 \mathrm{D}$ volume (B) had an $\mathrm{R}^{2}$-value of $46.4 \%$.
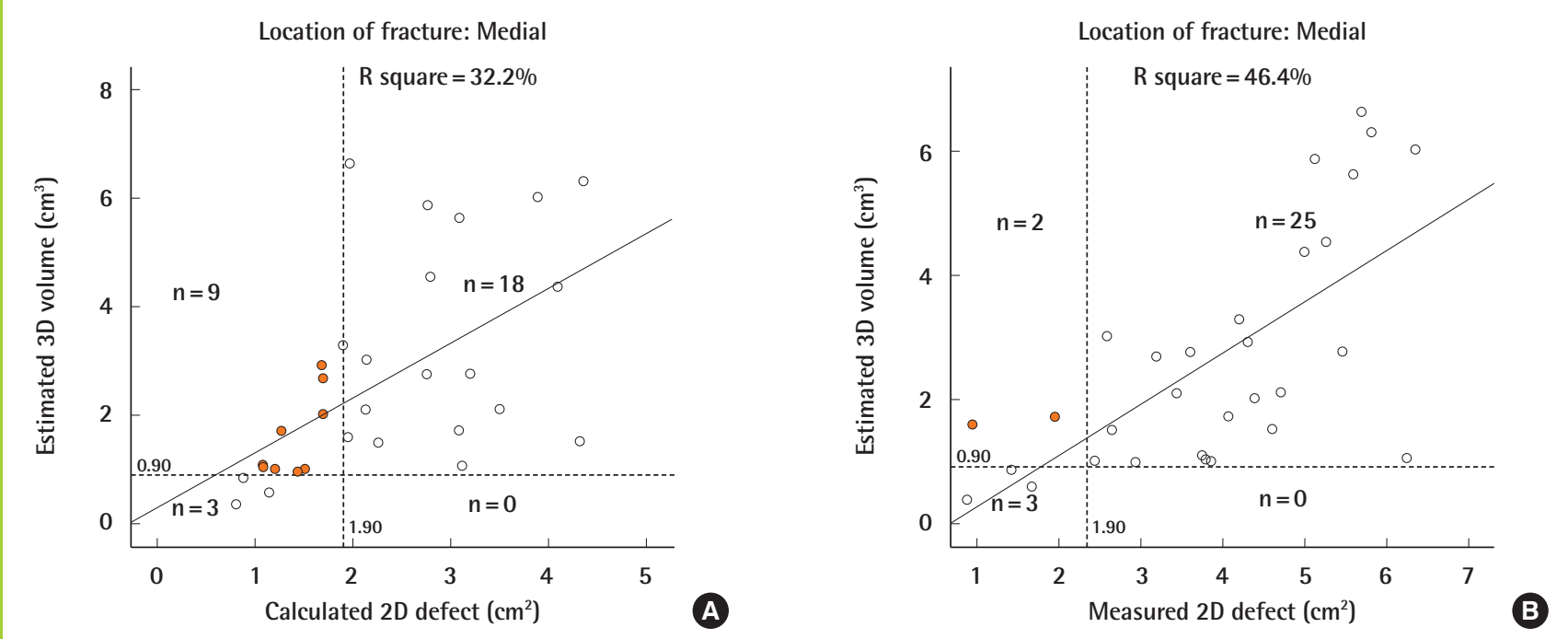

$\mathrm{mm})$. According to this criterion, 16 of the 30 patients in group I and 18 of the 30 patients in group $M$ had clinically large defects (with a calculated 2D extent greater than $1.9 \mathrm{~cm}^{2}$ ), and 25 of the 30 patients in group I and 27 of the 30 patients in group $\mathrm{M}$ showed a significant $3 \mathrm{D}$ herniated volume $\left(>0.9 \mathrm{~cm}^{3}\right)$ (Figs. $3 \mathrm{~A}, 4 \mathrm{~A})$. Based on the measured $2 \mathrm{D}$ extent, 26 of the 30 patients in group I and 25 of the 30 patients in group $\mathrm{M}$ had clinically large defects $\left(>1.9 \mathrm{~cm}^{2}\right)$, and 25 of the 30 patients in group I and 27 of the 30 patients in group M showed a signifi- cant 3D herniated volume $\left(>0.9 \mathrm{~cm}^{3}\right)$ (Figs. 3B, 4B).

\section{Correlation between the calculated or measured 2D} extent of the orbital defect and the estimated 3D volume of herniated orbital content

In group I, the correlation between the calculated 2D extent of the orbital defect and the 3D volume of the herniated orbital content was statistically significant, with a Pearson correlation coefficient of 0.788 and an $\mathrm{R}^{2}$-value of $62.1 \%$, and the corre- 
Table 3. Correlations between the 3-dimensional volume of herniated orbital content and the 2-dimensional extent of the defects

\begin{tabular}{|c|c|c|}
\hline Location of fracture & Pearson correlation & $\begin{array}{c}\text { 95\% Confidence } \\
\text { interval }\end{array}$ \\
\hline \multicolumn{3}{|l|}{ Group I $(n=30)$} \\
\hline Calculated 2D defect & $0.788^{\star *}$ & 0.565 to 0.884 \\
\hline Measured 2D defect & $0.739^{* *}$ & 0.499 to 0.862 \\
\hline \multicolumn{3}{|l|}{ Group M (n=30) } \\
\hline Calculated 2D defect & $0.568^{* *}$ & 0.261 to 0.770 \\
\hline Measured 2D defect & $0.682^{* *}$ & 0.426 to 0.836 \\
\hline
\end{tabular}

${ }^{* *} \mathrm{P}<0.05$.

sponding values for the correlation between the measured $2 \mathrm{D}$ extent and the 3D volume were 0.739 and $54.6 \%$, respectively $(\mathrm{P}<0.05)$ (Table 3).

In group $\mathrm{M}$, the correlation between the calculated $2 \mathrm{D}$ extent of the orbital defect and the 3D volume of the herniated orbital content was statistically significant, with a Pearson correlation coefficient of 0.568 and an $\mathrm{R}^{2}$-value of $32.2 \%$, and the corresponding values for the correlation between the measured $2 \mathrm{D}$ extent and the 3D volume were 0.682 and $46.4 \%$, respectively $(\mathrm{P}<0.05)$ (Table 3).

\section{DISCUSSION}

Most orbital fractures involve the medial orbital wall, orbital floor, and infraorbital groove and/or canal [16,17]. Conservative care is indicated for patients with good ocular motility and minimal displacement of the orbital content. On the contrary, surgical intervention should be considered for patients with a severely limited gaze, entrapment of the extraocular muscle, or predictable enophthalmos [6]. According to previous reports, enophthalmos of more than $2 \mathrm{~mm}$ may cause recognizable cosmetic problems [18]. In the early post-traumatic period, however, the degree of enophthalmos is difficult to predict due to confounding orbital edema. Therefore, it is crucial to predict the late development of enophthalmos in order to determine whether surgery is indicated [19].

Early studies investigating whether enophthalmos could be predicted focused on the $2 \mathrm{D}$ extent of the orbital defect, either calculated or measured. Manson et al. [2] reported that orbital defects of $2 \mathrm{~cm}^{2}$ could cause significant enophthalmos, while Sung et al. [7] showed that a defect of $2.75 \mathrm{~cm}^{2}$ could result in late enophthalmos of $2 \mathrm{~mm}$.

Since the computerized pixel-counting technique for orbital volume measurement was introduced by Bite et al. [10], subsequent reports have emphasized volumetric changes in attempts to predict the development of late enophthalmos. Whitehouse et al. [9] reported that a $1.0 \mathrm{~cm}^{3}$ increase in the orbital volume induced $0.77 \mathrm{~mm}$ of enophthalmos within 20 days after the initial injury. Fan et al. [5] reported a linear correlation between the increment of orbital volume and the degree of enophthalmos, with each $1.0 \mathrm{~cm}^{3}$ increment of orbital volume resulting in approximately $0.89 \mathrm{~mm}$ of enophthalmos. Recently, 3D processing software has been used for a variety of applications, including visualization, verification, measurements, analysis, customized implant design, rapid prototyping, linkage to $\mathrm{CAD}$ findings, and surgical simulations $[12,13,20]$.

However, no reports have elucidated the correlation between the $2 \mathrm{D}$ extent of orbital defects and the $3 \mathrm{D}$ volume of the herniated orbital content. We hypothesized that if a significant positive correlation is present between these 2 parameters, then each is clinically meaningful for predicting possible late enophthalmos and determining whether surgery is indicated.

Strongly positive correlations (Pearson correlation coefficient over 0.7) [21] between the calculated and measured 2D defect and 3D orbital volume of the herniated orbital contents in group I were found in this study (Pearson correlation coefficient, 0.788 and 0.739 , respectively; $\mathrm{P}<0.05$ ). A moderate positive correlation between the calculated and measured $2 \mathrm{D}$ extent of the defects and the 3D volume of the herniated orbital content in group $\mathrm{M}$ (Pearson correlation coefficient, 0.568 and 0.682 , respectively; $\mathrm{P}<0.05$ ) was noted. However, the $2 \mathrm{D}$ extent of the orbital defect was not sufficient to reflect the $3 \mathrm{D}$ herniated orbital volume protruding through the defect, as indicated by the low to moderate $\mathrm{R}^{2}$ values ranging from $32.2 \%$ to $62.1 \%$.

According to the criteria of Jin et al. [8], our study revealed that 10 of the 14 patients $(71.4 \%)$ in group I and 9 of the 12 patients (75\%) in group $\mathrm{M}$ who had clinically small defects (calculated $2 \mathrm{D}$ extent less than $\left.1.9 \mathrm{~cm}^{2}\right)$ showed a significant 3D volume of herniated content $\left(>0.9 \mathrm{~cm}^{3}\right)$. Additionally, 1 of the 4 patients (25\%) in group I and 2 of the 5 patients (40\%) in group $\mathrm{M}$ who had clinically small defects (measured 2D extent less than $1.9 \mathrm{~cm}^{2}$ ) showed similar results (Figs. 3, 4). These findings suggest that the $2 \mathrm{D}$ orbital defect-either calculated or measured - might not appropriately represent the herniated orbital volume. Therefore, the calculated or measured $2 \mathrm{D}$ extent of the orbital defect should be complemented with a $3 \mathrm{D}$ analysis of the volume of the protrusion to determine its clinical significance.

Since Deveci et al. [20] reported measurements obtained using a 3D software program as an easy and accurate method of assessing the volume of the orbit by making a comparison of direct measurements in 20 dry skulls, advanced 3D reconstructed imaging techniques have been introduced $[9,12,20]$. 
However, those studies estimated the 3D volume using a single-plane analysis with a reference plane on CT scans, and this technique was not able to avoid all potential sources of bias $[7,22]$. Kwon et al. [12] reported that measurements of the orbital volume using coronal CT scans underestimated the volume by $35.3 \%$ compared with the volume estimated using on the axial plane.

In this study, segmentation of the bony orbit and herniated soft tissue was performed using the interactive HU threshold interval in a semiautomatic manner. The physician selected the best threshold interval based on a visual analysis of the anatomic boundaries of the bony orbit and herniated orbital soft tissue. This technique is significantly faster and more reliable than manual segmentation and separation of herniated soft tissue from orbital bone. The mirror image of the uninjured bony orbit was used as a guideline to demarcate the boundary of the $3 \mathrm{D}$ volume on the defect surface. However, thin orbital walls may not be represented accurately in single CT scans. We applied 3D rendering models with fused multiplane radiologic data derived from the $\mathrm{CAD}$ software to overcome these drawbacks. Using $3 \mathrm{D}$ rendered multiplane images, we identified and traced the reconstructed orbital boundary to reduce measurement errors and to allow the accurate measurement of the $2 \mathrm{D}$ extent of the orbital defect and the 3D volume of the herniated content in orbital fractures.

Conventional orbital volume analysis has focused on determining the expansion ratio of the injured orbital volume compared with the uninjured orbital volume [2,5]. However, the normal variation between the volumes of the bilateral orbits has been reported to be as high as 7\%-8\% [23]. To minimize such volumetric differences, we traced the entire margin of the fractured orbit and then used the mirror image of the uninjured orbit to obtain the herniated orbital volume.

Our study classified orbital wall fractures into medial wall fractures and floor fractures. This classification was designed to account for the anatomical differences between the medial and inferior walls. Previous anatomical studies have shown that the medial wall is reinforced by the ethmoid air cells, whereas the orbital floor overlies the maxillary sinus, which has no reinforcing structures $[1,8,12]$. This may cause orbital wall fractures in the medial and inferior walls to be different, even in response to a similar trauma. Yano et al. [16] reported that punched-out fractures were the most frequent type of orbital blow-out fracture affecting both the medial wall and the floor, while the burst type occurred more often in the floor. Rhee et al. [24] reported that the punched-out fracture type showed a significant tendency to be larger than trap-door fractures in terms of both area and volume. These studies support the possibility that the relation- ship between the $2 \mathrm{D}$ extent of the defect and the $3 \mathrm{D}$ orbital volume could be different between these 2 groups because the different anatomy of these regions may lead to different fracture types.

Our study has some limitations. First, the 3D fused rendering model using multiplane $\mathrm{CT}$ scans may require expensive $3 \mathrm{D}$ processing software. Second, 2D and 3D measurements would be difficult to obtain in cases of extensive orbital wall fractures including the anatomical landmark of the orbital bony strut. Third, the orbital volume may have been overestimated because the CT scans were evaluated at the time of injury and the softtissue swelling had not yet fully subsided. Fourth, correlations of the $2 \mathrm{D}$ and $3 \mathrm{D}$ parameters with enophthalmos were not analyzed.

Nonetheless, our study is of significance, as it revealed that the 2D extent of orbital defects-either calculated or measuredwas not able to adequately represent the $3 \mathrm{D}$ volume of the herniated orbital content protruding through the orbital defect. Therefore, combining an assessment of the $2 \mathrm{D}$ extent of the orbital defect with an estimation of the 3D volume of the herniated orbital content would be helpful in determining whether surgery is indicated and in ensuring satisfactory surgical outcomes in procedures performed to treat isolated orbital wall fractures.

\section{REFERENCES}

1. Burm JS, Chung CH, Oh SJ. Pure orbital blowout fracture: new concepts and importance of medial orbital blowout fracture. Plast Reconstr Surg 1999;103:1839-49.

2. Manson PN, Grivas A, Rosenbaum A, et al. Studies on enophthalmos: II. The measurement of orbital injuries and their treatment by quantitative computed tomography. Plast Reconstr Surg 1986;77:203-14.

3. Park MS, Kim YJ, Kim H, et al. Prevalence of diplopia and extraocular movement limitation according to the location of isolated pure blowout fractures. Arch Plast Surg 2012;39: 204-8.

4. Hwang K, Huan F, Hwang PJ. Diplopia and enophthalmos in blowout fractures. J Craniofac Surg 2012;23:1077-82.

5. Fan X, Li J, Zhu J, et al. Computer-assisted orbital volume measurement in the surgical correction of late enophthalmos caused by blowout fractures. Ophthal Plast Reconstr Surg 2003;19:207-11.

6. Burnstine MA. Clinical recommendations for repair of isolated orbital floor fractures: an evidence-based analysis. Ophthalmology 2002;109:1207-10.

7. Sung YS, Chung CM, Hong IP. The correlation between the degree of enophthalmos and the extent of fracture in medial 
orbital wall fracture left untreated for over six months: a retrospective analysis of 81 cases at a single institution. Arch Plast Surg 2013;40:335-40.

8. Jin HR, Shin SO, Choo MJ, et al. Relationship between the extent of fracture and the degree of enophthalmos in isolated blowout fractures of the medial orbital wall. J Oral Maxillofac Surg 2000;58:617-20.

9. Whitehouse RW, Batterbury M, Jackson A, et al. Prediction of enophthalmos by computed tomography after 'blow out' orbital fracture. Br J Ophthalmol 1994;78:618-20.

10. Bite U, Jackson IT, Forbes GS, et al. Orbital volume measurements in enophthalmos using three-dimensional CT imaging. Plast Reconstr Surg 1985;75:502-8.

11. Lee WT, Kim HK, Chung SM. Relationship between smallsize medial orbital wall fracture and late enophthalmos. J Craniofac Surg 2009;20:75-80.

12. Kwon J, Barrera JE, Most SP. Comparative computation of orbital volume from axial and coronal CT using three-dimensional image analysis. Ophthal Plast Reconstr Surg 2010;26:26-9.

13. Weissheimer A, Menezes LM, Sameshima GT, et al. Imaging software accuracy for 3-dimensional analysis of the upper airway. Am J Orthod Dentofacial Orthop 2012;142: 801-13.

14. Feeman TG. The mathematics of medical imaging: a beginner's guide. New York: Springer; 2010.

15. Ploder O, Klug C, Backfrieder W, et al. 2D- and 3D-based measurements of orbital floor fractures from CT scans. J Craniomaxillofac Surg 2002;30:153-9.
16. Yano H, Nakano M, Anraku K, et al. A consecutive case review of orbital blowout fractures and recommendations for comprehensive management. Plast Reconstr Surg 2009; 124:602-11.

17. Choi KE, Lee J, Lee H, et al. The paradoxical predominance of medial wall injuries in blowout fracture. J Craniofac Surg 2015;26:e752-5.

18. Koo L, Hatton MP, Rubin PA. When is enophthalmos "significant"? Ophthal Plast Reconstr Surg 2006;22:274-7.

19. Park MS, Baek S. Measurement of fracture size using the picture archiving communication system in an outpatient clinic for factors that influence postoperative enophthalmos in adult inferior orbital wall fractures. J Craniofac Surg 2013; 24:1692-4.

20. Deveci M, Ozturk S, Sengezer M, et al. Measurement of orbital volume by a 3-dimensional software program: an experimental study. J Oral Maxillofac Surg 2000;58:645-8.

21. Hinkle DE, Wiersma W, Jurs SG. Applied statistics for the behavioral sciences. Sth ed. Boston: Houghton Mifflin Company; 2003.

22. Ploder O, Klug C, Voracek M, et al. A computer-based method for calculation of orbital floor fractures from coronal computed tomography scans. J Oral Maxillofac Surg 2001;59:1437-42.

23. Parsons GS, Mathog RH. Orbital wall and volume relationships. Arch Otolaryngol Head Neck Surg 1988;114:743-7.

24. Rhee SH, Kim TS, Song JM, et al. Effectiveness of computed tomography for blow-out fracture. Maxillofac Plast Reconstr Surg 2014;36:273-9. 